\title{
Correction: Cao, Z., et al. The Expression and Functional Significance of Runx2 in Hepatocellular Carcinoma: Its Role in Vasculogenic Mimicry and Epithelial-Mesenchymal Transition. Int. J. Mol. Sci. 2017, 18, 500
}

\author{
Zi Cao ${ }^{1}$, Baocun Sun ${ }^{1,2,3, *}$, Xiulan Zhao ${ }^{1,2}$, Yanhui Zhang ${ }^{3}$, Qiang Gu ${ }^{1,2}$, Xiaohui Liang ${ }^{1}$, Xueyi Dong ${ }^{1}$ \\ and Nan Zhao ${ }^{1}$
}

check for updates

Citation: Cao, Z.; Sun, B.; Zhao, X.; Zhang, Y.; Gu, Q.; Liang, X.; Dong, X.; Zhao, N. Correction: Cao, Z., et al. The Expression and Functional Significance of Runx2 in Hepatocellular Carcinoma: Its Role in Vasculogenic Mimicry and Epithelial-Mesenchymal Transition. Int. J. Mol. Sci. 2017, 18, 500. Int. J. Mol. Sci. 2021, 22, 77. https://dx.doi.org/10.3390/ijms2201 0077

Received: 8 December 2020

Accepted: 15 December 2020

Published: 23 December 2020

Publisher's Note: MDPI stays neutral with regard to jurisdictional claims in published maps and institutional affiliations.

Copyright: (C) 2020 by the authors. Licensee MDPI, Basel, Switzerland. This article is an open access article distributed under the terms and conditions of the Creative Commons Attribution (CC BY) license (https: / / creativecommons.org/ licenses/by/4.0/).
1 Department of Pathology, Tianjin Medical University, Tianjin 300070, China; imcaozi@163.com (Z.C.); xiulanzhao@aliyun.com (X.Z.); wyft1022@163.com (Q.G.); liangxiaohui123@126.com (X.L.); dxy7235202@126.com (X.D.); zhaonantj@tmu.edu.cn (N.Z.)

2 Department of Pathology, General Hospital of Tianjin Medical University, Tianjin 300052, China

3 Department of Pathology, Cancer Hospital of Tianjin Medical University, Tianjin 300060, China; Yanhuizhang015@163.com

* Correspondence: baocunsun@aliyun.com; Tel./Fax: +86-022-8333-6813

The author wishes to make the following corrections to this paper [1]. The reasons for the corrections are: (1) error in representing Negative expression of Runx2 immunohistochemical staining picture in the old version of Figure 1, the mistake was due to mixing up the Negative expression of Runx2 immunohistochemical staining picture with the Negative expression of VE-cadherin immunohistochemical staining picture in the old version of Figure 1, it should be replaced with the correct Negative expression of Runx2 immunohistochemical staining pictures in the new version of Figure 1; (2) error in representing DAPI-stained SMMC7721-shRunx2-LGALS3 cell nuclei picture in the old version of Figure 6, the mistake was due to mixing up DAPI-stained SMMC7721-shRunx2-LGALS3 cell nuclei picture with DAPI-stained SMMC7721 cell nuclei picture, it should be replaced with the correct DAPI-stained SMMC7721-shRunx2-LGALS3 cell nuclei picture in the new version of Figure 6.

The correction does not change the conclusions of this manuscript. The authors would like to apologize for any inconvenience caused to the readers by these changes. 


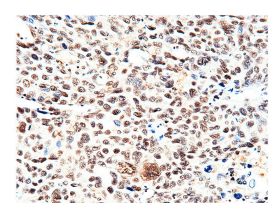

A

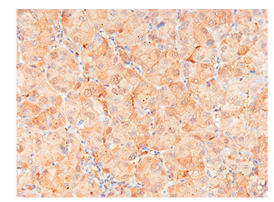

E

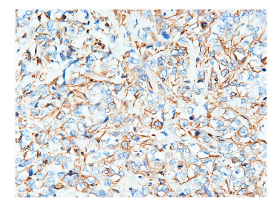

100um $\quad$ ।

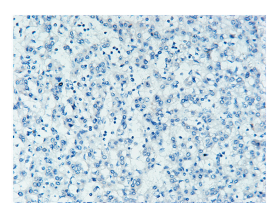

B

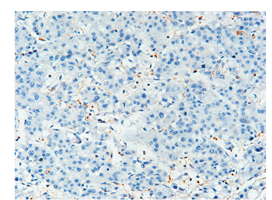

$\mathrm{F}$

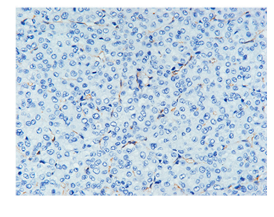

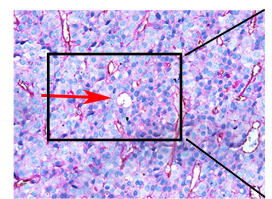

C

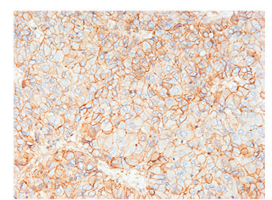

G

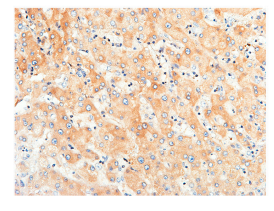

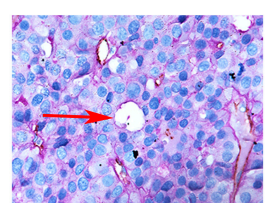

D

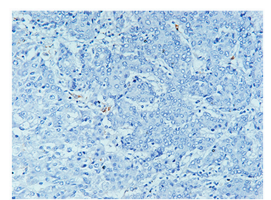

$\mathrm{H}$

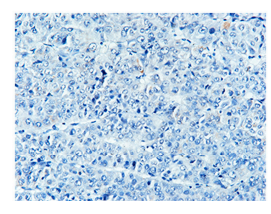

$\mathrm{L}$

Figure 1. Hepatocellular carcinoma specimens were analyzed by immunohistochemistry. (A) Runx2 was predominantly localized in the nuclear of cancer cells $(\times 200$; bars $100 \mu \mathrm{m})$; (B) Negative expression of Runx2 $(\times 200$; bars $100 \mu \mathrm{m})$; (C) CD31/PAS double staining displayed VM channels (Red arrow) in Hepatocellular carcinoma specimens $(\times 200 ;$ bars $100 \mu \mathrm{m})$; (D) VM channels (Red arrow) $(\times 400$; bars $100 \mu \mathrm{m})$; (E) Nuclear and cytoplasmic staining of Galectin-3 $(\times 200$; bars $100 \mu \mathrm{m})$; (F) Negative expression of Galectin-3 $(\times 200$; bars $100 \mu \mathrm{m})$; (G) Positive E-cadherin expression $(\times 200$; bars $100 \mu \mathrm{m})$; $(\mathbf{H})$ Negative E-cadherin expression $(\times 200$; bars $100 \mu \mathrm{m})$; (I) Positive Vimentin expression $(\times 200$; bars $100 \mu \mathrm{m})$; (J) Negative Vimentin expression $(\times 200$; bars $100 \mu \mathrm{m}) ;(\mathbf{K})$ Positive VE-cadherin expression $(\times 200 ;$ bars $100 \mu \mathrm{m})$; (L) Negative VE-cadherin expression $(\times 200$; bars $100 \mu \mathrm{m})$.

VE-cadherin

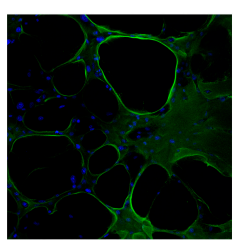

A

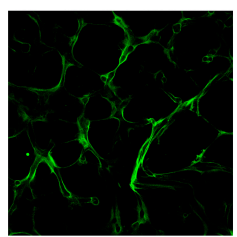

D

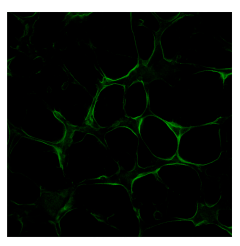

100um $\quad G$
DAPI

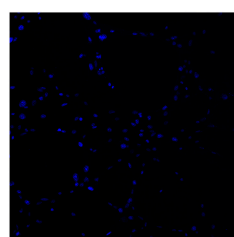

B

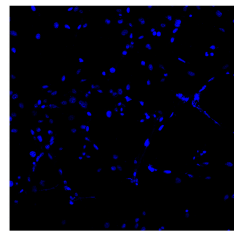

E

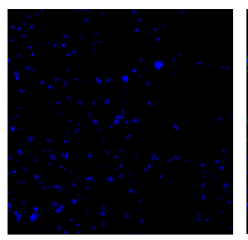

H
Merge

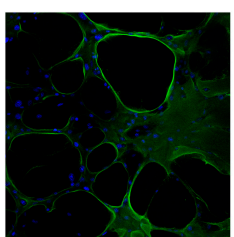

c

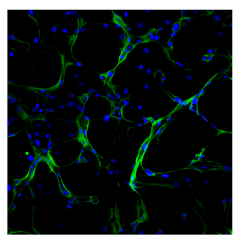

F

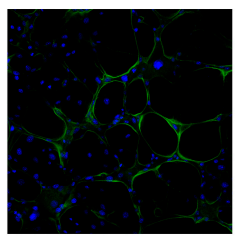

I

Figure 6. The VM-like tubes formed by HepG2-Runx2 cells, SMMC7721 cells and SMMC7721shRunx2-LGALS3 cells were assessed by VE-cadherin immunofluorescence and confocal microscopy $(\times 200)$. (A,D,G) VE-cadherin staining of the VM channel was concentrated in the wall of the tubes; $(\mathbf{B}, \mathbf{E}, \mathbf{H})$ The cell nuclei were stained by DAPI; $(\mathbf{C}, \mathbf{F}, \mathbf{I})$ The merged images showed that the expression of VE-cadherin in VM networks. 
Funding: This research received no external funding.

Conflicts of Interest: The authors declare no conflict of interest.

\section{Reference}

1. Cao, Z.; Sun, B.; Zhao, X.; Zhang, Y.; Gu, Q.; Liang, X.; Dong, X.; Zhao, N. The Expression and Functional Significance of Runx2 in Hepatocellular Carcinoma: Its Role in Vasculogenic Mimicry and Epithelial-Mesenchymal Transition. Int. J. Mol. Sci. 2017, 18, 500. [CrossRef] [PubMed] 